\title{
The role of coxI-associated repeated sequences in plant mitochondrial DNA rearrangements and radish cytoplasmic male sterility
}

\author{
Christopher A. Makaroff ${ }^{1}$, Ingrid J. Apel ${ }^{2}$, and Jeffrey D. Palmer ${ }^{3}$ \\ 1 Department of Chemistry, Miami University, Oxford, OH 45056, USA \\ 2 Department of Internal Medicine, University of Michigan Medical School, Ann Arbor, MI 48109, USA \\ 3 Department of Biology, Indiana University, Bloomington, IN 47405, USA
}

Received August 10, 1990

\begin{abstract}
Summary. The gene coxI, encoding subunit I of mitochondrial cytochrome $\mathrm{c}$ oxidase, has been characterized from the normal (fertile) and Ogura (male-sterile) cytoplasms of radish to determine if a previously identified mitochondrial DNA rearrangement, and its associated transcriptional differences, could play a role in Ogura cytoplasmic male sterility (CMS). The normal and Ogura loci are virtually identical for $2.8 \mathrm{~kb}$, including a 527 codon open reading frame whose product is approximately $95 \%$ identical to other plant COXI polypeptides. A rearrangement $120 \mathrm{bp} 5^{\prime}$ to the coding region results in different $5^{\prime}$ transcript termini for the two genes. A comparison of several crucifer mitochondrial DNAs indicates that this rearrangement also occurs in the normal radish cytoplasm and is, therefore, not involved in Ogura CMS. Sequences present at the coxI locus belong to at least two different dispersed repeat families, members of which are also associated with other rearranged genes in radish.
\end{abstract}

Key words: Brassica - Mitochondrial DNA - Cytoplasmic male sterility - Recombination

\section{Introduction}

In contrast to the small and conserved structure of animal mitochondrial genomes, plant mitochondrial DNAs (mtDNAs) are large in size $(200-2500 \mathrm{~kb})$ and complex in sequence organization (Gray 1989). With the exception of Brassica hirta (Palmer and Herbon 1987), all plant mtDNAs characterized thus far exhibit a complex multipartite organization in which a circular master chromosome, which contains a complete complement of genetic information, is resolved into smaller subgenomic circles via high-frequency recombination across one or more pairs of directly repeated sequences. Although plant $m t D N A s$ contain a large number of repeated sequences, which vary in size from 55 bp (Rottmann et al. 1987) to

Offprint requests to: C. A. Makaroff
$14 \mathrm{~kb}$ (Lonsdale et al. 1984), not all of them appear to be active in high-frequency intramolecular recombination. For example, at least six families of repeat elements have been identified in the radish genome, yet only the largest is active in high-frequency recombination (Makaroff and Palmer, unpublished). It has been suggested that the high degree of structural variability observed among plant mtDNAs of different species may be the result of low-frequency recombination events across repeated sequences dispersed throughout the genome (Palmer and Herbon 1988; Lonsdale et al. 1988). These low-frequency recombination events, which occasionally become fixed in the mitochondrial population, have been implicated in the generation of mutants with altered mitochondrial function (Dewey et al. 1986; Hanson et al. 1988; Makaroff et al. 1989).

Cytoplasmic male sterility (CMS), a maternally inherited trait that results in the inability of a plant to produce functional pollen, is one class of mutations in which the consequences of mtDNA rearrangements on mitochondrial function can be studied (Lonsdale 1987; Newton 1988). There are many examples of mtDNA rearrangements and altered transcriptional and translational patterns associated with male-sterile plants. The mitochondrial genome of the male-sterile cytoplasm of Ogura radish, which is highly rearranged relative to its normal (fertile) counterpart, is one system in which mtDNA rearrangements have been associated with altered transcript patterns (Makaroff and Palmer 1988). Specifically, altered trancript patterns have been identified for three genes: atp $A$, atp6, and coxI. We present here the characterization of coxI from both normal and Ogura radish cytoplasms in order to examine the effects of mitochondrial DNA rearrangements on gene expression and CMS.

\section{Materials and methods}

Plant material. Raphanus sativus cv. Scarlet Knight (wild-type radish) was the source of the normal radish cytoplasm. The mitochondrial genome of Scarlet Knight exhibits the same restriction enzyme profile as those of three other commonly used male-fertile 
radish cultivars (Palmer 1988; Makaroff and Palmer, unpublished) and thus is used to represent what we term the "normal" cytoplasm of radish. The rapid-cycling radish line CrGC15 (R1rr; obtained from the Crucifer Genetics Cooperative) was the source of the Ogura cytoplasm. Sterile and fertile (nuclear-restored) plants containing the Ogura cytoplasm were distinguished by their ability to produce functional pollen. B. campestris cv. Purple Top White Globe, $B$. hirta (commercial white mustard line) and B. nigra (commercial black mustard line) were also used for mtDNA comparisons.

Isolation of nucleic acids. Plasmid DNA was isolated by the alkaline lysis procedure (Birnboim and Doly 1979) and purified by $\mathrm{CsCl}$ gradients where necessary. Mitochondrial DNA was isolated from leaves of 6 week-old plants by the DNase I procedure (Kolodner and Tewari 1972). Mitochondrial RNA (mtRNA) was isolated in the presence of aurintricarboxylic acid (Stern and Newton 1986) from mitochondria purified by differential centrifugation.

Sequence determination and analysis. Normal and Ogura coxI were previously isolated as $P_{s t} \mathrm{I} 9.0 \mathrm{~kb}$ and $S a l \mathrm{I} 15.0 \mathrm{~kb}$ clones in pUC8 and pTZ18, respectively (Palmer and Herbon 1986; Makaroff and Palmer 1988). The two genes and their flanking sequences were then subcloned as Pst I-SaII3.7 kb (normal radish) and SacI-Sall $4.8 \mathrm{~kb}$ (Ogura radish) fragments in pTZ18 and Bluescript SK +, respectively. DNA sequencing was conducted using the dideoxy chain termination procedure (Sanger et al. 1977) on clones generated by either the exonuclease III technique (Henikoff 1984) or by restriction enzyme digestion. Four synthetic oligonucleotides were synthesized using an Applied Biosystems DNA synthesizer (Foster City, CA) and used to prime DNA synthesis. DNA sequences were analyzed using the Eugene program (Department of Cell Biology, Baylor College of Medicine, Houston, Tex. 77030).

Southern analysis of coxI and flanking regions. Subclones containing various segments of normal and Ogura coxI loci were hybridized to Southern blots containing restriction digests (PstI, SalI and KpnI) of normal and Ogura radish mtDNAs (see Makaroff and Palmer 1988 for restriction fragment profiles and detailed maps of the two genomes), as well as single digests (Pst $\mathrm{I})$ of $B$. campestris, Ogura radish, normal radish, $B$. hirta and $B$. nigra mtDNAs. Methods used for restriction endonuclease digestion, agarose gel electrophoresis, bidirectional transfer from agarose gels to nylon membranes, and labeling of plasmid DNA by nick-translation, were as described previously (Maniatis et al. 1982; Palmer 1986).

Nuclease mapping. DNA probes were labeled with either $\left[\alpha^{32} \mathrm{P}\right]-$ dATP, by primer extension of single-stranded DNA using the Klenow fragment of DNA polymerase I, or $\left[\gamma^{32} \mathrm{P}\right]$-ATP with T4 polynucleotide kinase, and then purified by phenol extraction, polyacrylamide gel electrophoresis, electroelution and ethanol precipitation prior to use in nuclease mapping experiments. Transcript map ping experiments were conducted using previously published procedures (Berk and Sharp 1978) essentially as described by Makaroff et al. (1989). Protected fragments were sized on $5 \%$ non-denaturing polyacrylamide gels next to ${ }^{32} \mathrm{P}$-labeled Bluescript $\mathrm{KS}+$ digested with $\operatorname{HinFI}$, and also on high-resolution $6 \%$ polyacrylamide $8 \mathrm{M}$ urea gels next to dideoxy sequencing ladders.

\section{Results}

\section{The normal radish coxI locus}

Normal radish coxI was subcloned and sequenced as a $3.7 \mathrm{~kb}$ PstI-SalI fragment. A $1581 \mathrm{bp}$ open reading frame, capable of encoding a 527 AA polypeptide, is identified as coxI on the basis of DNA sequence identity (Fig. 1). The derived amino acid sequence of radish coxI exhibits between 94 and $97 \%$ identity with the deduced polypeptides from sorghum (Bailey-Serres et al. 1986), soybean (Grabau 1986), wheat (Bonen et al. 1987), Oenothera (Hiesel et al. 1987) and maize (Isaac et al. 1985). However, if the coxI transcripts are edited, as has been observed for several other plant mitochondrial genes (Covello and Gray 1989; Gualberto et al. 1989; Schuster et al. 1990), then we would expect the amount of amino acid similarity to be different from calculations based on DNA sequence analysis.

Two different repeat elements are present at the coxI locus. From nucleotides -118 to 133 there is a $249 \mathrm{bp}$ sequence (belonging to repeat family $\mathrm{B}$ ) that is duplicated $97 \mathrm{bp}$ downstream of the atp6 coding region in normal radish (Makaroff et al. 1989). The two sequences are $99.6 \%$ identical. This sequence maps to the $3^{\prime}$ rearrangement breakpoint of the normal and Ogura atp 6 genes and, based on Southern hybridization experiments (Fig. 2A), appears to be present at least four and five times in the normal radish and Ogura radish mtDNAs, respectively.

Also present in the $5^{\prime}$ flanking region of coxI is a $688 \mathrm{bp}$ sequence (nucleotides -882 to -194 ) that is repeated in an inverted orientation upstream of normal radish atp A (Makaroff et al. 1990). The two sequences are $98.4 \%$ identical. This region is also present upstream of Ogura atp $A$ but, because of a rearrangement, Ogura atp $A$ contains only $445 \mathrm{bp}$ of this repeat (belonging to repeat family A). A portion of this region (nucleotides -595 to -420 ) is also found immediately upstream of orf 105 at the Ogura atp6 locus (Makaroff et al. 1989). Southern hybridizations, using a $219 \mathrm{bp}$ fragment ( -675 to -456$)$ internal to the repeat, indicate that this sequence is present, in part, at least four times in normal radish and six times in Ogura radish (Figs. $2 \mathrm{~A}$ and 5).

Because the only copy of coxI (designated coxI-1) in the predominant (major) mitochondrial genome of normal radish is present on a $20.5 \mathrm{~kb}$ Pst I fragment

Fig. 1. Nucleotide and derived amino acid sequences of the coxI loci from normal and Ogura radish. The Ogura sequence (nucleotides-1955 to 2862 ; top line) is aligned with the normal radish coxI-2 locus (nucleotides -886 to 2860 ; bottom line), a form that is present in low copy number. Where the sequences are identical, only the coxI-2 sequence is shown. Insertions/deletions relative to coxI-2 are represented by $(-)$. Numbering of nucleotides is from the predicted translation start of coxI. A tobacco cpDNA-homologous sequence in Ogura radish is overlined (nucleotides - 1932 to -1333). Regions homologous to sequences present at the atp $A$ ( $A$; nucleotides -882 to -194$)$ and normal radish atp6 $(B$; nucleotides -118 to 133 ) loci are underlined. A 175 bp sequence (nucleotides -595 to -420 ) present at the Ogura orf105-atp6 locus, which is also present inpart upstream of the tobacco atp 6 and rps13 genes and Oenothera coxI, coxII and atp6 genes and Petunia coxII, is overlined with a dashed line. Major $5^{\prime}$ transcript termini are indicated with horizontal arrows and $3^{\prime}$ transcript termini are indicated with vertical arrowheads (above the sequence for Ogura radish and below the sequence for normal radish) 


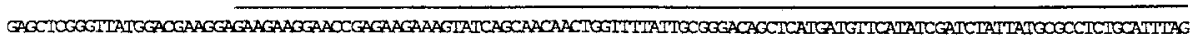

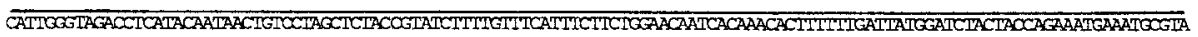

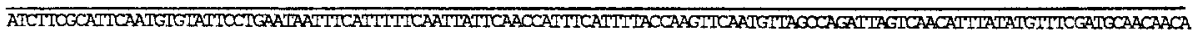

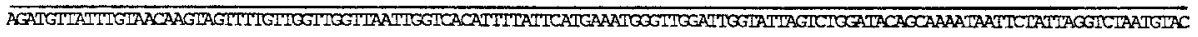

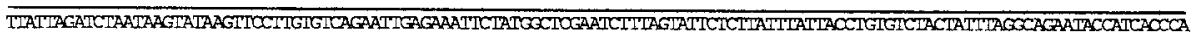

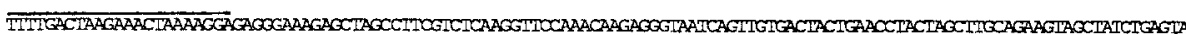

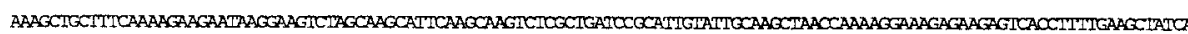

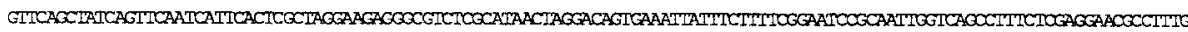

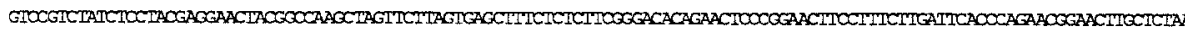
CIGCAgG

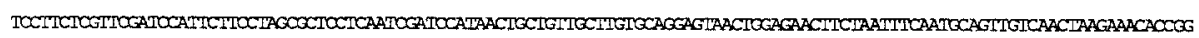

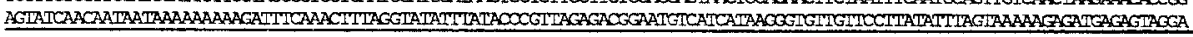

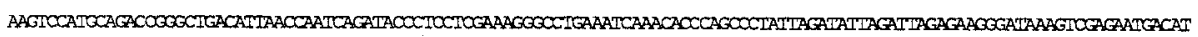

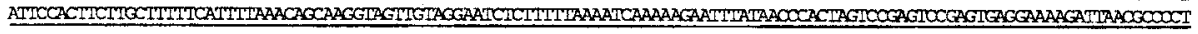

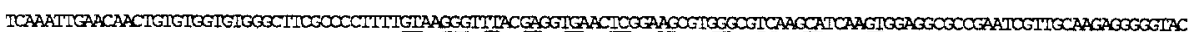

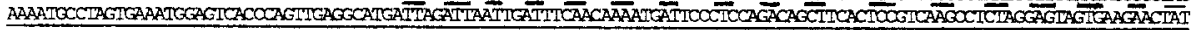

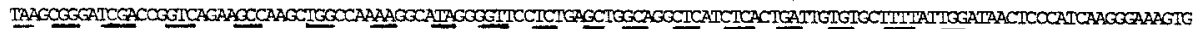

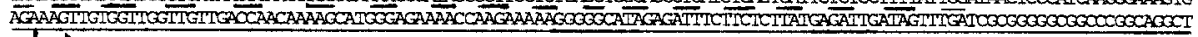

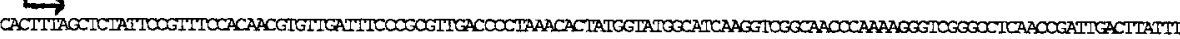

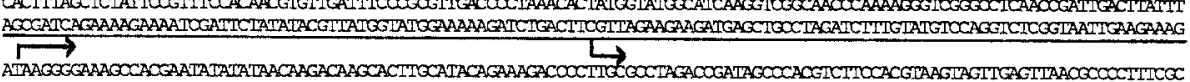

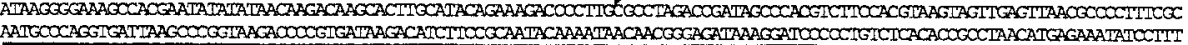
AACAAGISCICCAGICACTCCGACAAACOOCTITIOAA A

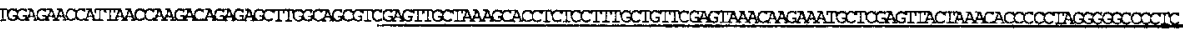

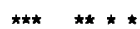
C B

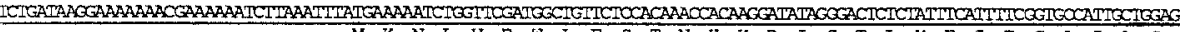

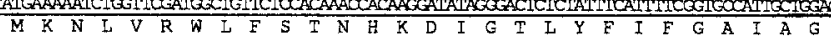

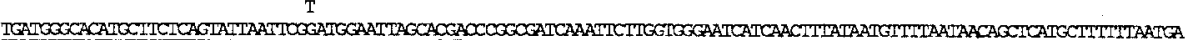

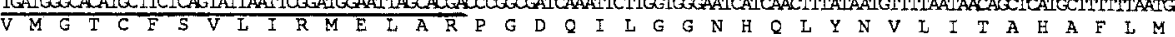

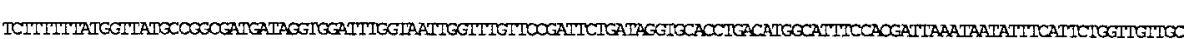

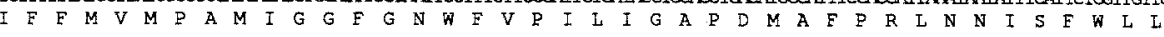

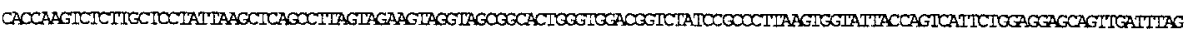
$P$ P S I L L L L L L S S S A L L V

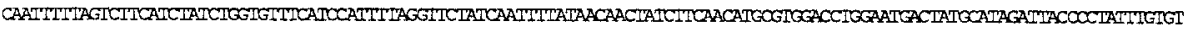

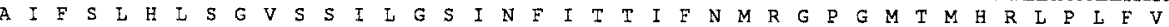

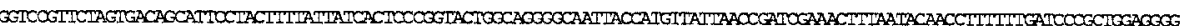

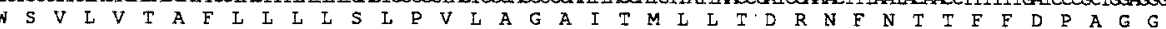

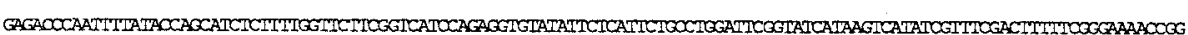

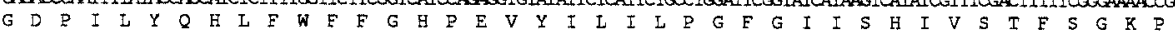

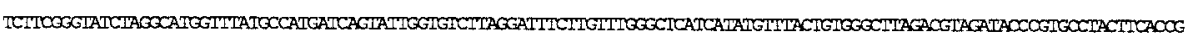

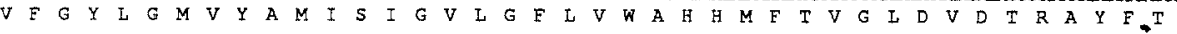

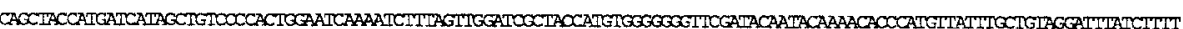

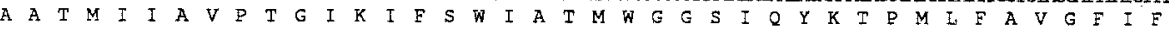

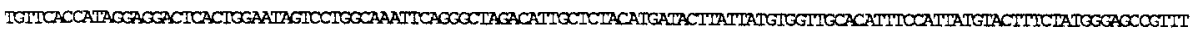

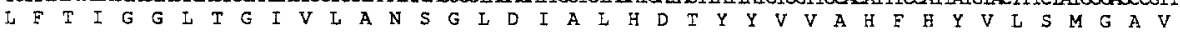

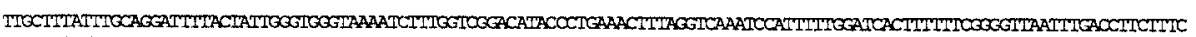

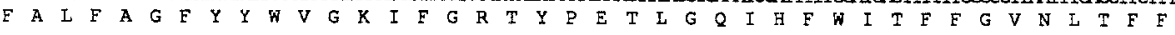

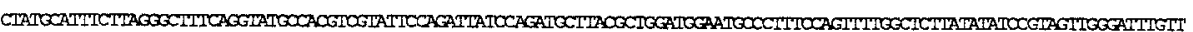
$P$ M H F L G L S G M P R R I P D Y P D A Y A G W N A I S S F G S Y I S V V G I C GIIICITCGIGGICGTAACAATCACTITAACCAGIGGAAATAACAAAAGATGIGCIOCAAGICCTTGOSCTCTTCAACTTAATTCAACTPCACTGGAATGGATGGTACAAAGTCCIOCTG

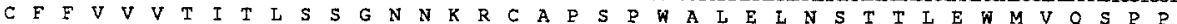

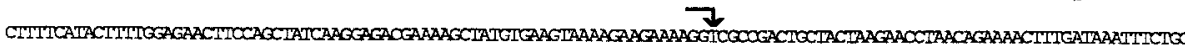
A F H T F G E L P A I K E T K S Y V K

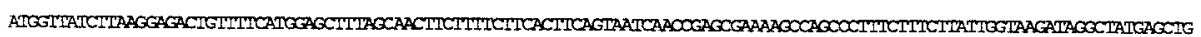
TAGGACIGCACTGAGATGGCAGATGAGAGAT- AGIGCAAAACG

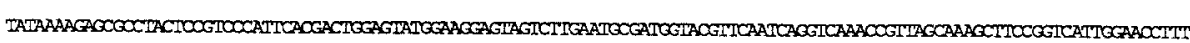

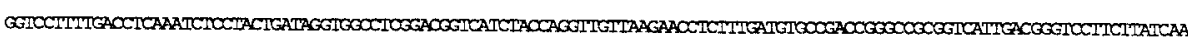

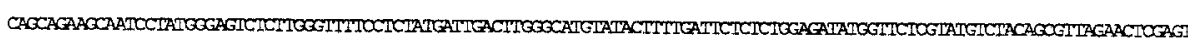

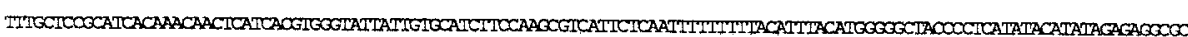

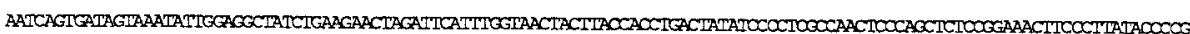

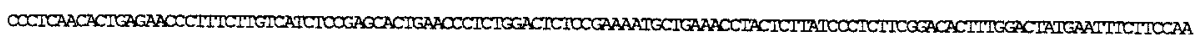

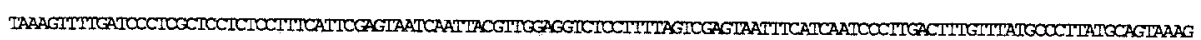

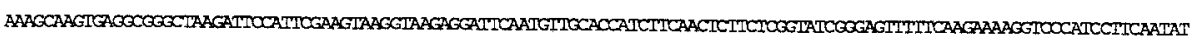
ATEATIGGGIOCAC 


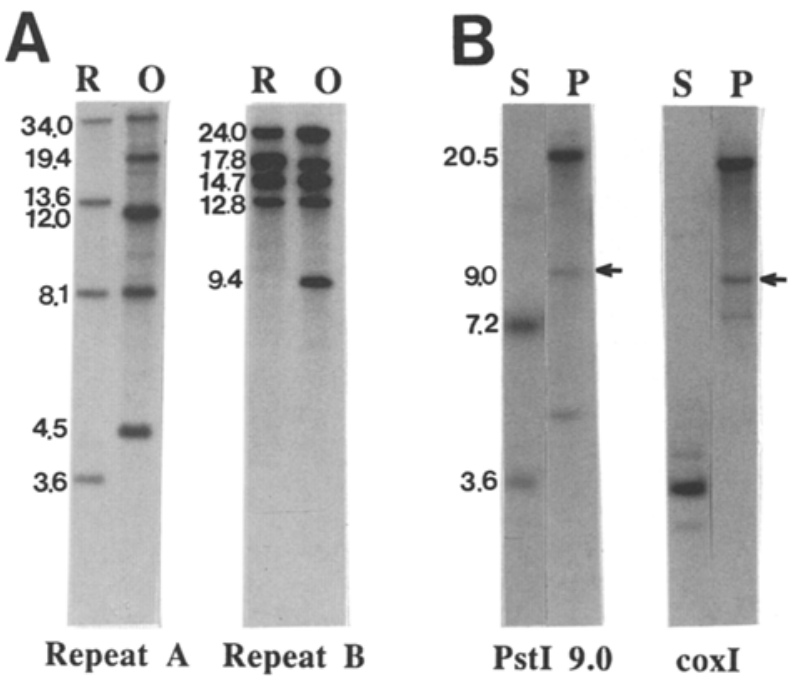

Fig. 2A, B. Southern hybridization analysis of coxI repeat elements and the Pst $\mathrm{I} 9.0 \mathrm{~kb}$ fragment. A hybridization of a $219 \mathrm{bp}$ segment of repeat $A$ (nucleotides -459 to -678 ) and a $442 \mathrm{bp}$ fragment containing repeat B2 (nucleotides 2111 to 2553; Makaroff et al. 1989) from the atp6 locus to Southern blots containing either $S a l \mathbb{I}$ (repeat A) or $K p n I$ (repeat B) restriction digests of normal radish $(R)$ and Ogura $(O)$ mtDNA. B Southern blots containing $S a l I(S)$ and $P_{s t} \mathrm{I}(\mathrm{P})$ digests of normal radish mtDNA probed with the normal radish $P$ st $\mathrm{I} 9.0 \mathrm{~kb}$ fragment (which carries coxI-2), and a $2.9 \mathrm{~kb}$ fragment containing the coxI-coding and 3 '-flanking regions. Sizes of fragments are indicated in $\mathrm{kb}$ to the left of the autoradiographs. The submolar $P_{s t} \mathrm{I} 9.0 \mathrm{~kb}$ fragment is denoted with an arrow

(Makaroff and Palmer 1988), and because the gene (designated $\operatorname{cox}(-2)$ we characterized was originally isolated as a $9.0 \mathrm{~kb}$ PstI fragment (Palmer and Herbon 1986), the relationship of the Pst $9.0 \mathrm{~kb}$ clone to the $20.5 \mathrm{~kb}$ fragment was determined. The predominant mitochondrial genome of normal radish contains two $9.1 \mathrm{~kb}$ Pst $\mathrm{I}$ fragments, neither of which is identified in Southern hybridization experiments using a coxI probe (data not shown). The $9.0 \mathrm{~kb}$ PstI fragment hybridizes strongly to normal radish $S a l$ fragments of 3.6 and $7.2 \mathrm{~kb}$ (Fig. 2B), which are largely contained within the $20.5 \mathrm{~kb} P s t \mathrm{I}$ fragment. No hybridization is detected to an adjacent $30.3 \mathrm{~kb}$ SalI fragment, which contains approximately $11 \mathrm{~kb}$ of the $P_{s t} \mathrm{I} 20.5$ fragment and no other strong hybridization signals from other regions of the genome were detected. This indicates that the Pst $\mathrm{I} 9.0 \mathrm{~kb}$ fragment is derived from the left half of the $P_{s t} \mathrm{I} 20.5 \mathrm{~kb}$ fragment. Further characterization of the Pst $\mathrm{I} 9.0 \mathrm{~kb}$ fragment indicates that it is present at submolar levels in the normal radish mitochondrion and that it was generated by recombination across a second copy of repeat $\mathrm{A}$ that is present $5^{\prime}$ to atp $A$. DNA sequence and Southern blot analyses indicate that the two normal radish coxI loci are virtually identical from nucleotide -615 to +2860 (Makaroff, Apel and Palmer, unpublished). Because the two normal radish coxI loci (coxI-1 and coxI-2) both contain the same region of the locus that defines the Ogura/normal radish rearrangement breakpoint, (see below) the normal radish coxI-2/Ogura radish coxI alignment should provide an accurate comparison of the two loci.

\section{The Ogura radish coxI locus}

Ogura coxI, previously localized to a $S a l I 15.0 \mathrm{~kb}$ clone (Makaroff and Palmer 1988), was subcloned and sequenced as a $S a l l-S a c \mathrm{I} 4.8 \mathrm{~kb}$ fragment. The Ogura sequence is shown aligned with the normal radish coxI-2 sequence in Fig. 1. The Ogura coxI and normal radish coxI-2 sequences are virtually identical $(99.9 \%$ sequence identity at aligned positions) from nucleotide -118 to the $3^{\prime}$-end of the sequence. Two nucleotide substitutions (positions -7 and 117) and one insertion/deletion (positions 1797-1 806) were identified. The nucleotide difference at position 117 occurs in the coding region but is silent (CGG and CGT both code for arginine). From the $5^{\prime}$-end of the sequenced regions to nucleotide -118 the sequences are completely different, indicating that a rearrangement occurred at or after nucleotide -117 . The exact site of recombination cannot be determined because it occurred within a repeated sequence. Repeat B5 (249 bp) begins at nucleotide - 117, suggesting that it was involved in both the coxI and $3^{\prime}$ atp6 (Makaroff et al. 1989) rearrangements that have occurred between normal and Ogura radish.

Located between nucleotides -1932 and -1331 in the Ogura locus is a 601 bp sequence that exhibits $94 \%$ identity to the inverted repeat of tobacco cpDNA (nucleotides 111178 to 111772 and nucleotides 130876 to 131351; Shinozaki et al. 1986). This sequence corresponds to internal segments of tobacco chloroplast ORF350 and ORF1244 (amino acids 54 to 267). The reading frame of the mitochondrial sequence is unaltered relative to the chloroplast reading frames despite $33 \mathrm{nu}-$ cleotide differences, which result in 15 amino acid changes, and a 6-bp insertion/deletion. Examination of the nucleotide sequences around the two radish mtDNA integration borders failed to identify any common sequence, or notable secondary structure, that would provide information on the mechanism of the integration process. Transcripts from this region were not detected in Northern blots of mtRNA isolated from radish storage roots (data not shown), consistent with our earlier finding that mtRNAs corresponding to cpDNA-homologous sequences present in B. campestris mtDNA are, in general, not detectable (Makaroff and Palmer 1987). In addition, this sequence is present in all crucifer mtDNAs examined to date (Nugent and Palmer 1988) and is, therefore, unlikely to be related to CMS.

\section{Transcription of coxI genes}

Nuclease-mapping experiments using a 685 bp normal radish fragment (nucleotides -879 to -194 ), end-labeled on the coding-strand, produced abundant protected fragments of 319 and 142 nucleotides and low levels of fully protected probe (Fig. 3). These represent one major $5^{\prime}$ transcript terminus at -513 and multiple transcript termini centered at -336 . A probe originating internal to the coxIcoding region identified the same transcript termini as the BamHI-labeled probe (data not shown), indicating that the observed protected fragments are from the coxI locus 


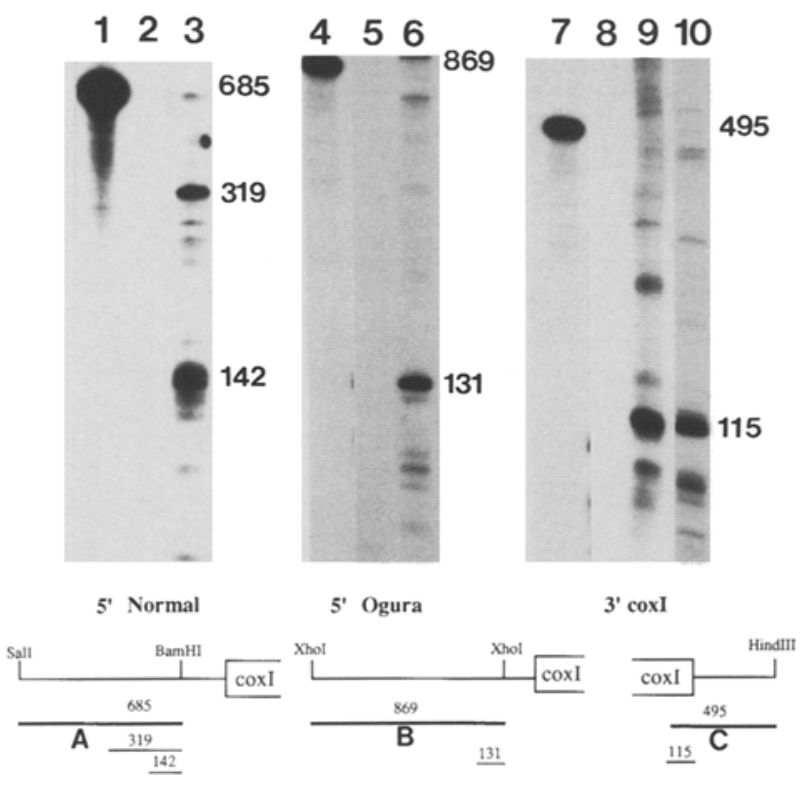

Fig. 3. Nuclease mapping of the transcript termini for normal and Ogura radish coxI. Top: autoradiographs of nuclease mapping of normal (lanes $1-3$ and $7-9$ ) and Ogura (lanes $4-6$ and 10) radish using probes shown below. Lane 1 , probe $A$; lane 2 , probe $A$ plus $20 \mu \mathrm{g}$ of yeast $\mathrm{tRNA}$ treated with S1 nuclease; lane 3 , probe $A$ plus $10 \mu \mathrm{g}$ of normal radish mtRNA treated with $\mathrm{S} 1$ nuclease; lane 4: probe $B$; lane 5 , probe $B$ plus $20 \mu$ g of yeast tRNA treated with S1 nuclease; lane 6 , probe $B$ plus $10 \mu \mathrm{g}$ of Ogura radish mtRNA treated with $\mathrm{S} 1$ nuclease; lane 7 , probe $C$; lane 8 , probe $C$ plus $20 \mu \mathrm{g}$ yeast tRNA treated with $\mathrm{S} 1$ nuclease; lane 9 , probe $C$ plus $10 \mu \mathrm{g}$ normal radish $\mathrm{mtRNA}$ treated with $\mathrm{S} 1$ nuclease; lane 10 , probe $C$ plus $10 \mu \mathrm{g}$ of Ogura radish mtRNA treated with $\mathrm{S} 1$ nuclease. The sizes of the probes and abundant protected fragments are shown in bp to the right of each panel. Bottom: schematic representation of probes used in nuclease mapping experiments and the resulting most-abundant protected fragments. Probe $A$ (heavy line) was a 691 bp fragment from the $5^{\prime}$ end of normal radish coxI- 1 end-labeled at the $B a m H I$ site. Protected fragments (thin lines) of 319 and 142 nucleotides were detected with probe $A$. Probe $B$ (heavy line) was an 869 nucleotide, uniformily-labeled, fragment corresponding to the coding-strand of Ogura radish coxI. A protected fragment of 131 nucleotides was obtained with probe $B$. Probe $C$ was a 495 nucleotide fragment from the 3 '-end of normal radish coxI that was uniformly labeled as the complement of the coxI mRNA by primer extension. A protected fragment of 115 nucleotides was obtained from both normal and Ogura radish mtRNA with probe $C$. The normal and Ogura radish sequences are identical in this region of the locus

and are not the result of hybridization to repeated sequences from elsewhere in the genome. Sequences showing limited similarity to those found at some putative plant mtRNA-processing sites (Young et al. 1986) are present near the shorter transcript but not the larger one. The $3^{\prime}$ end of the normal radish coxI transcript was mapped using a 495 nucleotide, uniformly-labeled probe (nucleotides 1482 to 1977). An abundant protected fragment of 115 nucleotides, as well as several low-abundance fragments, was obtained (Fig. 3). This corresponds to a major $3^{\prime}$ transcript terminus at +1597 . Transcript termini at -336 and $-513\left(5^{\prime}\right)$ and $+1597\left(3^{\prime}\right)$ predict transcripts of 1933 and 2110 . These correspond well with the observed coxI transcripts of $1950 \mathrm{nt}$ and $2150 \mathrm{nt}$ (Makaroff and Palmer 1988).

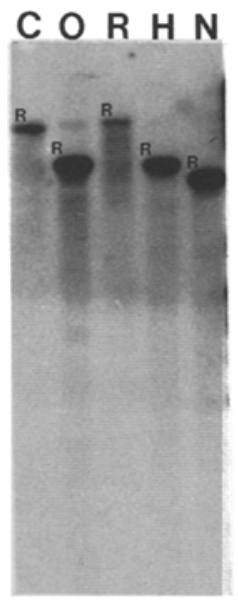

Radish

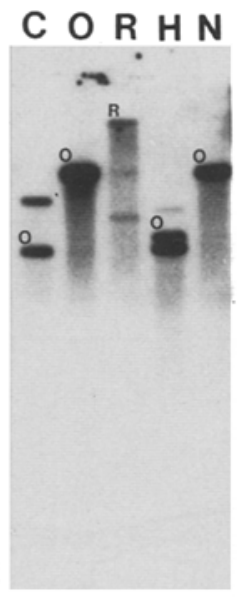

3' coxl

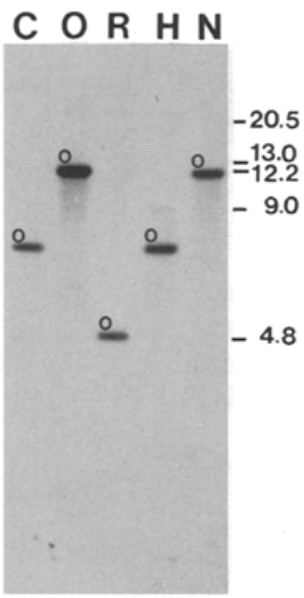

Ogura
Fig. 4. Determination of the origin of the coxI rearangement. Southern blot analysis of the coxI locus in $B$. campestris $(C)$, Ogura radish $(O)$, normal radish $(R), B$. hirta $(H)$ and $B$. nigra $(N)$. The hybridization probes were as follows: $477 \mathrm{bp} \mathrm{BamHI/Sall}$ fragment $5^{\prime}$ normal radish coxI-1; coxI coding region and $3^{\prime}$-flanking region (nucleotides 1080 to 2860 ); $5^{\prime}$ Ogura radish fragment (nucleotides -1911 to -142 ). Fragments that hybridize to the normal radish $5^{\prime}$ coxI-1 probe are denoted by $(R)$; those that hybridize to the Ogura radish $5^{\prime}$ cox $I$ probe by $(O)$. Size are shown in $\mathrm{kb}$

Nuclease mapping experiments to determine the $5^{\prime}$ terminus of Ogura coxI using an 869 nucleotide, uniformly-labeled probe extending from nucleotides -1101 to -143 resulted in a protected fragment of 131 nucleotides (Fig. 3). This corresponds to a $5^{\prime}$ transcript terminus at -274 . This site maps to a 9 bp region that exhibits sequence identity with the $16 \mathrm{bp}$ plant mtRNA processing site consensus sequence proposed by Young et al. (1986). No sequence identity was found between either the Ogura or normal radish $5^{\prime}$ transcript termini and a $50 \mathrm{bp}$ consensus sequence present at the 5-termini of abundant short transcripts from several plant mitochondrial genes that exhibit complex transcript patterns (Schuster and Brennicke 1989). The $3^{\prime}$ end of the Ogura coxI transcript was mapped using a uniformly-labeled 495 nucleotide fragment (nucleotides 1482 to 1977 ). An abundant 115 nucleotide-protected fragment was obtained (Fig. 3) which corresponds to a $3^{\prime}$ transcript terminus at +1597 . This maps to the same site as that identified for the $3^{\prime}$ transcript terminus of normal radish coxI and indicates that the observed difference in the coxI transcript patterns (Makaroff and Palmer 1988) is due solely to the $5^{\prime}$ rearrangement.

\section{Genomic organization of the coxI loci}

Southern hybridization analysis was used to resolve whether the coxI rearrangement occurred in normal or Ogura radish, and also to determine the relative position and orientation of the coxI sequences in the normal and Ogura radish genomes. Clones containing the coxI-cod- 


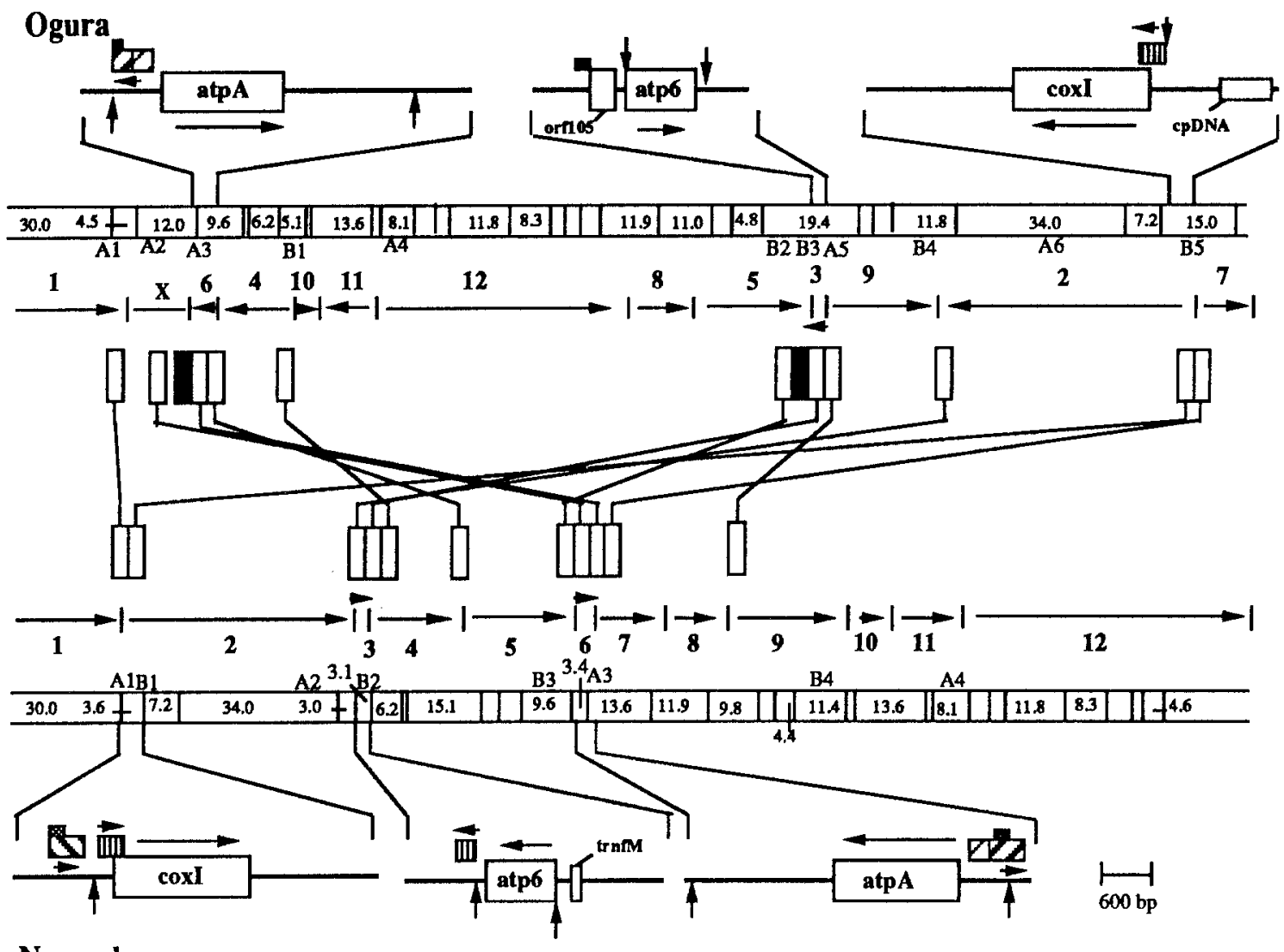

\section{Normal}

Fig. 5. Sall restriction map of the master chromosome and schematic representation of the atpA, atp 6 and coxI loci in the mitochondrial genomes of Ogura (top) and normal (bottom) radish. The relative arrangement of homologous sequences in the two genomes is shown below (Ogura) and above (normal) the restriction maps. Numbers and arrows indicate the positions and relative orientations of blocks of cross-hybridizing sequences whose arrangement has been conserved between the two genomes. Crossing lines connect homologous sequence blocks (rectangles) that are found at one of three loci (atpA, atp 6 or $\operatorname{cox} I)$ in either of the two radish genomes. Solid rectangles represent sequences not found in normal radish.

ing and $3^{\prime}$-flanking regions, as well as the normal and Ogura radish coxI $5^{\prime}$-flanking regions, were used to probe Southern blots containing $P_{s t} \mathrm{I}$ digests of mtDNAs from normal radish, Ogura radish, B. campestris, B. hirta and $B$. nigra (Fig. 4). The Ogura coxI 5'-flanking region probe hybridizes to the same fragments as the coxI-coding region probe in the three Brassica mtDNAs, indicating that the two sequences are closely linked in the four mitochondrial genomes. The coxI 5'-flanking region of normal radish hybridizes to the same fragment as the coding region probe in normal radish only, indicating that the sequences from the normal radish coxI $5^{\prime}$-flanking and coding regions are present at different locations in each of the three Brassica mitochondrial genomes. This indicates that the $5^{\prime}$ arrangement of the coxI gene in the three Brassica species is identical to that of Ogura radish and different from that of normal radish. We conclude, therefore, that the coxI rearrangment occurred in normal radish and that this locus is most likely not involved in Ogura radish CMS. Hybridization of the coxI-coding and $3^{\prime}$-flanking region to two fragments in the $B$. campes-
Heavy lines above (Ogura) and below (normal) represent three loci, atp $A$, atp 6 , and coxI, that have been characterized by DNA sequence analysis. Vertical arrows indicate rearrangement breakpoints; horizontal arrows above or below the genes indicate the direction of transcription. Letters below and above the Ogura and normal radish restriction maps, respectively, indicate additional fragments that exhibit weak cross-hybridization with the repeats (labeled $A$, boxes shaded with diagonal lines and $B$, boxes shaded with vertical lines). Data summarized in this figure are from Makaroff and Palmer 11988; Makaroff et al. 1989 and 1990; this paper and our unpublished results

tris and $B$. hirta genomes suggests that rearrangements have also occurred $3^{\prime}$ to coxI in these two genomes.

\section{Discussion}

\section{CMS and coxI alterations}

We have conducted a detailed analysis of the Ogura and normal radish coxI loci to determine if previously identified mtDNA rearrangements, and altered transcript patterns, associated with the coxI gene (Makaroff and Palmer 1988) are directly related to the Ogura CMS phenotype. Two different lines of evidence suggest that coxI plays no role in Ogura CMS: (1) The observed differences in the structure of the two loci and their transcript patterns are the result of a single rearrangement that occurred $119 \mathrm{bp} 5^{\prime}$ to the normal radish coxI coding region. No differences in the predicted coxI polypeptides were detected, nor were any novel genes generated by the rearrangement. (2) A comparison of the organization of coxI 
in the two radish mtDNAs and three Brassica mtDNAs indicates that the rearrangement occurred in the lineage leading to normal radish and not in the Ogura genome. While rearrangements $3^{\prime}$ to coxI have been identified in several Brassica mtDNAs (Palmer and Herbon 1988), the mtDNAs of $B$. campestris, $B$. hirta and $B$. nigra all share the Ogura radish arrangement of the coxI 5'-flanking region.

Mitochondrial DNA rearrangments have also been found at the coxI locus in the 9E cytoplasm of male-sterile sorghum (Bailey-Serres et al. 1986). In this case a rearrangement occurred in the cox $I$-coding region and results in the synthesis of a modified form of the protein. However, it appears that the cytochrome $c$ oxidase activity of mitochondria isolated from seedlings containing the $9 \mathrm{E}$ cytoplasm is not affected by this modification (Leaver et al. 1988). These results, along with our data on radish coxI, indicate that many of the numerous transcriptional and possibly translational differences observed for mitochondria isolated from CMS plants are the result of unrelated $\mathrm{mtDNA}$ rearrangements and have no causal role in CMS.

\section{Repeated sequences and radish $m t D N A$ rearrangements}

The coxI locus contains two different repeated sequences that appear to have been involved in genomic rearrangements. Figure 5 shows the locations of these coxI-associated repeated sequences relative to rearrangement events characterized for three genes (atpA, atp 6 and $c o x I$ ) in the normal and Ogura radish mitochondrial genomes. A 249 bp repeat (repeat family B) is present $3^{\prime}$ to the normal/Ogura coxI rearrangement breakpoint and also $3^{\prime}$ to the 3'-atp6 rearrangement breakpoint in normal radish. There are at least two and four additional copies of this repeat in the normal and Ogura mitochondrial genomes, respectively.

A second larger repeat (repeat family A), present six and four times in the Ogura and normal radish genomes respectively, is implicated in the normal radish coxI-1/ coxI-2 and normal/Ogura atpA rearrangements (Makaroff et al. 1990). Present within this repeat is a $122 \mathrm{bp}$ sequence that exhibits $90 \%$ sequence identity with a $175 \mathrm{bp}$ sequence common to the $5^{\prime}$-flanking regions of tobacco atp6 (Bland et al. 1987) and rps13 (Bland et al. 1986). This sequence, present upstream of the orf105-atp6 locus in Ogura radish (Makaroff et al. 1989), normal radish atp A (Makaroff et al. 1990), Petunia coxII (Pruitt and Hanson 1989) and the Oenothera coxI, coxIII and atp6 genes (Hiesel et al., 1987; Schuster and Brennicke 1987 ), is found in the same orientation relative to its associated gene in all cases except for the normal radish coxI locus. This repeat is included in the transcripts of all the above genes except radish atp $A$. While the function of the 122 bp repeat is unknown, its presence in the $5^{\prime}$-flanking sequences of genes from several diverse plants raises the possibility that it may serve as some type of regulatory element.

Although some sequences are found repeated at several different radish rearrangement breakpoints, no sin- gle sequence is universally present. This suggests that the rearrangements are due to general homologous recombination and not to a site-specific process. However, because the two radish genomes are so highly rearranged, such that individual rearrangement events cannot be traced, the possibility that a specific sequence is required either $3^{\prime}$ or $5^{\prime}$ to the site of recombination cannot be eliminated.

While it is now clear that repeated sequences are involved in rearrangement processes that result in high levels of within-plant mtDNA heterogeneity and betweenline mtDNA diversity, many questions remain to be answered about the origin and maintenance of repeats, the systems involved in the recombination process, and the effect of rearrangement on mitochondrial function. Answers to these questions should be obtained through the continued analysis of recombinant plant mitochondrial genomes and alterations associated with CMS, through studying the intensified recombination and rearrangement occurring in some cultured plant cell lines and somatic hybrids, and by introducing defined repeats into mitochondrial genomes via transformation, once this approach becomes feasible.

Acknowledgements. This work was supported by grants RO1GM35087 (JDP) and F32-GM11323 (CAM) from the National Institutes of Health and DMB-8919264 (CAM) from the National Science foundation. We thank P. Calie and M. Kushel for critical reading of the manuscript and $D$. Bay for photographic services.

\section{References}

Bailey-Serres J, Hanson DK, Fox TD, Leaver CJ (1986) Cell 47:567-576

Berk AJ, Sharp PA (1978) Cell 12: 721-732

Birnboim HC, Doly J (1979) Proc Natl Acad Sci USA 7:1513-1523

Bland MM, Levings CS III, Matzinger DF (1986) Mol Gen Genet 204:8-16

Bland MM, Levings CS III, Matzinger DF (1987) Curr Genet 12: $475-481$

Bonen L, Boer PM, McIntosh JE, Gray MW (1987) Nucleic Acids Res 15:6734

Covello PS, Gray MW (1989) Nature 341:662-666

Dewey RE, Levings CS III, Timothy DH (1986) Cell 44:439-449

Gray MW (1989) Annu Rev Cell Biol 5:25-56

Grabau EA (1986) Plant Mol Biol 7:377-384

Gualberto JM, Lamaltenia L, Bonnard G, Weil J-H, Grienenberger JM (1989) Nature 341:660-662

Hanson MR, Young EG, Rothenberg M (1988) Phil Trans R Soc Lond B 319:199-208

Henikoff S (1984) Gene 28:351-359

Hiesel R, Schobel W, Schuster W, Brennicke A (1987) EMBO J $6: 29-34$

Isaac PG, Jones VP, Leaver CJ (1985) EMBO J 4:617-1623

Kolodner R, Tewari KK (1972) Proc Natl Acad Sci USA 69:18301834

Leaver CJ, Isaac PG, Small ID, Bailey-Serres J, Liddell AD, Hawkesford MJ (1988) Phil Trans R Soc Lond B 319:165-176

Lonsdale LM (1987) Plant Physiol Biochem 25:265-271

Lonsdale DM, Hodge TP, Fauron CM-R (1984) Nucleic Acids Res 12:9249-9261

Lonsdale DM, Brears T, Hodge TP, Melville SE, Rottman WH (1988) Phil Trans R Soc Lond B 319:149-163

Maniatis T, Fritsch EF, Sambrook J (1982) Molecular cloning A laboratory manual, Cold Spring Harbor Laboratory, Cold Spring Harbor, New York, pp 107-147 
Makaroff CA, Palmer JD (1987) Nucleic Acids Res 15:5141-5156 Makaroff CA, Palmer JD (1988) Mol Cell Biol 8:1474-1480

Makaroff CA, Apel IJ, Palmer JD (1989) J Biol Chem 264:1170611713

Makaroff CA, Apel IJ, Palmer JD (1990) Plant Mol Biol (in press) Newton K (1988) Annu Rev Plant Physiol Plant Mol Biol 39:503-532 Nugent JM, Palmer JD (1988) Curr Genet 14:501-509

Palmer JD (1986) Methods Enzymol 118:167-186

Palmer JD (1988) Genetics 118:341-351

Palmer JD, Herbon LA (1986) Nucleic Acids Res 14:9755-9765

Palmer JD, Herbon LA (1987) Curr Genet 11:565-570

Palmer JD, Herbon LA (1988) J Mol Evol 28:87-97

Pruit KD, Hanson MR (1989) Curr Genet 16:281-291

Rottmann WH, Brears T, Hodge TP, Lonsdale DM (1987) EMBO J $6: 1541-1546$
Sanger F, Nicklen S, Coulson AR (1977) Proc Natl Acad Sci USA 74: 5463

Schuster W, Brennicke A (1987) Nucleic Acids Res 15:9092

Schuster W, Brennicke A (1989) Curr Genet 15:187-192

Schuster W, Wissinger B, Unseld M, Brennicke A (1990) EMBO J 9:263-269

Shinozaki K, Ohme M, Tanaka M, Wakasugi T, Hayashida N, Matsubayashi T, Zaita N, Chunwongse J, Obokata J, Yamaguchi-Shinozaki K, Ohto C, Torazawa K, Meng BY, Sugita M, Deno H, Kamogashira T, Yamada K, Kusuda J, Takaiwa F, Kato A, Tohdoh N, Shimada H, Sugiura M (1986) EMBO J 5:2043-2049

Stern DB, Newton KJ (1986) Methods Enzymol 118:488-496

Young EG, Hanson MR, Dierks PM (1986) Nucleic Acids Res $14: 7995-8006$ 